\title{
In Vitro Performance Testing of Nanoparticulate Drug Products for Parenteral Administration
}

\author{
Elena Fecioru ${ }^{1,2}$, Martin Klein ${ }^{1}$, Johannes Krämer ${ }^{3}$, and Matthias G. Wacker ${ }^{4 *}$ \\ ${ }^{1}$ PHAST Development GmbH \& Co. KG, Konstanz, Germany. \\ 2Institute of Pharmaceutical Technology, Goethe University, Frankfurt am Main, Germany. \\ ${ }^{3} \mathrm{DISSO} \mathrm{GmbH}$, Homburg, Germany. \\ ${ }^{4}$ Department of Pharmacy, National University of Singapore, Singapore.
}

e-mail: phamgw@nus.edu.sg

\begin{abstract}
The parenteral administration route is most effective for the delivery of drug substances with poor oral bioavailability. Nanoparticulate drug delivery systems were developed with the specific purpose of overcoming the obstacles met by conventional drug therapy for parenteral administration in case of drug targeting or passing biobarriers. Today, in vitro performance tests are a prerequisite for formulation development and quality control of nanoparticulate dosage forms, focusing on drug release and stability of the release rate in particular if the release should extend to days. As a consequence of the diverse technologies of these novel dosage forms, at present there is no standardized in vitro release test available. With a focus on parenterally administered formulations, this review describes published methods used for in vitro drug release testing of nanoparticulate drug products. Some of the techniques may be applicable to nanoparticulate dosage forms for oral application.
\end{abstract}

KEYWORDS: Dissolution testing, nanoparticulate drug products, parenteral administration, dispersion releaser, fiber optic system

\section{INTRODUCTION}

oday, conventional drug therapies using the parenteral administration route include sterile solutions, emulsions, suspensions, and implants (13). They involve a number of challenges such as decreased physical or chemical stability of excipients and drugs in aqueous solutions, poor drug solubility and absorption to macromolecules at the site of administration, degradation by enzymes, limited access to the site of action, as well as the need for a suitable controlled/sustained drug release system. To overcome some of these drawbacks, nanoscaled drug products are being used (2). These complex systems can be categorized according to their carrier principle and composition of the carrier. They can be manufactured through multiple techniques, which can be divided in two main categories: top-down and bottomup (4-7). Drug substances can either be entrapped in liposomes, solid lipid nanoparticles, or polymeric nanoparticles or bound to their surface (8). Nanocarrier systems are usually administered parenterally in the form of a liquid dispersion (2). Together with the formulation technology, the site of administration will dictate the release of the drug substance. Thus, intravenous (IV) administration is preferred when immediate systemic drug availability and rapid onset of action is desired or when physiological barriers prevent access to the target site, as is the case of the blood-brain barrier (BBB), whereas subcutaneous or intramuscular administration will ensure long-term drug delivery by forming a depot (9). Besides the carrier-based systems for small molecules, nanoparticulate drug products containing biological drug substances and nano-crystals or combinations thereof exist, but are not discussed in this review.

Nanotechnology-related drug products may present specific physicochemical properties due to the large surface to volume ratio and may have the ability to actively target a specific tissue, improve solubility of poorly soluble substances, control or sustain the drug release, and/or extend systemic elimination (10-13). Compared to conventional drug products, the small particle diameter results in a release mechanism controlled by drug-carrier 
interaction rather than by diffusion. As a consequence, the drug and excipients properties are of major importance for the therapeutic success.

Drug targeting strategies enable the nanoparticles to overcome physiological barriers such as the BBB (14, 15). By elevating the drug concentration in the central nervous system, brain diseases can be treated more effectively. Further, by modifying the particle surface with targeting ligands, specific cells within the human body can be addressed (16). In a study involving doxorubicin loaded poly(lactide-co-glycolide) (PLGA) nanoparticles, controlling formulation parameters such as coating, type of polymer, drug substance, and stabilizer enabled efficient brain delivery of doxorubicin for the treatment of intracranial 101/8 glioblastoma in rats (17). The nanoparticulate formulation developed by Gelperina et al. provided promising results when administered for the first time in humans (17).

Although a clear definition unanimously accepted by academia, regulatory bodies, and the pharmaceutical industry is yet to be established, recommendations on the nomenclature of nanoparticles have been made by the European Commission, the U.S. Food and Drug Administration (FDA), and the Standardization Administration of China (18-20). According to the U.S. FDA, nanoparticles may be defined as manufactured particles in the size range of 1-100 nm or even outside this range, up to $1000 \mathrm{~nm}$, if they exhibit specific properties that can be directly attributed to their dimension (21).

In the context of an increasing need for regulatory requirements for nanoparticulate drugs, regulatory bodies from around the world are striving for a consensus in defining nanoparticles (22). The U.S. FDA issued a draft guidance for industry, which includes a general overview on the topic of in vitro release testing of drug products that contain nanomaterials (21). Furthermore, the European Medicines Agency co-sponsored an international regulators expert group involving the US, Japan, Canada and Australia, and organized the First International Scientific Workshop on Nanomedicines followed by the International Pharmaceutical Regulators Forum (IPRF) platform for scientific exchange $(23,24)$.

On this background, well established characterization methods and standardized in vitro release testing methods for nanotechnology-related drug products represent a prerequisite for formulation development and quality control. In the case of nanoparticulate drug products designed for controlled or sustained release purposes, the drug substance is usually entrapped in the core matrix, from where it is slowly released. Thus, the term in vitro release testing can be used for performance testing of nanoparticulate drug products (25). Numerous methods have been described in the literature for testing in vitro release from nanoparticulate drug products, among which membrane diffusion methods, sampling and separation methods, and continuous flow methods are most frequently employed (26-28). This review article intends to give an overview of current concepts and methods for testing in vitro release of parenteral nanoparticulate drug products, describing suitability and limitations of the present methodology.

\section{PHYSIOLOGICAL CONDITIONS AT THE SITE OF DRUG RELEASE}

Once nanosuspensions are injected into the blood stream, the surface properties and the size of the nanoparticle are the main parameters that influence the fate of the nanoparticulate drug in the system. These are a result of the formulation process, usually affecting the biological interactions. For nanoparticulate drug products designed to circulate longer and accumulate in a specific tissue, a wide variety of size ranges have been reported. Depending on the material and charge, nanoparticles with a size between 10 and $500 \mathrm{~nm}$ are able to circulate in the human capillary system (10). They are cleared by liver and the mononuclear phagocyte system much slower as compared to those below $10 \mathrm{~nm}$, which face a more rapid clearance through the kidneys (10). Nanoparticulate drugs present in the bloodstream can interact with opsonins (antibodies, complement proteins, and circulating proteins), which tend to adsorb on the nanoparticles due to their surface properties. As a result, agglomeration of the nanoparticles can take place, which leads to modifications of the drug release rates or to their elimination by the reticuloendothelial system (RES) (29, 30). By coating the surface of the nanoparticulate drug product with block copolymers, such as polyethylene glycol (PEG), agglomeration and fast elimination by the RES can be avoided. Nanoparticulate drug products that went through the process of surface coating with hydrophilic polymers are called "stealth nano carriers," as is the case of stealth liposomes (for example, doxorubicin [Doxil, Johnson \& Johnson, USA]) (12, 30-32).

When designing an in vitro release test, physiological factors first have to be taken into consideration. During method development, it is preferred for the in vitro test conditions to replace the more complex physiology mimicking media by simple and robust in vitro media, with ingredients reduced to the ones being essential for drug release. Further, the in vitro setting should reflect

|Dissolution 
the mechanism of drug release occurring in the in vivo situation. If systemic action is desired, the onset of release may take place in the blood. For efficient target delivery, most of the release is usually intended to take place at the site of action.

For example, the central nervous system is available for release of the drug substance only after the nanoparticulate drug has passed the BBB. In addition to blood, the medium in which release is supposed to occur is the cerebrospinal fluid (CSF). Compared to the blood, the volume of medium available for drug release is $\mathbf{1 5 0}$ $\mathrm{mL}$ of CSF. Differences in composition between CSF and blood plasma regarding $\mathrm{pH}$, protein content, glucose, and ionic concentration are presented in Table 1 . In vivo, these two compartments are separated by the BBB (33). The mechanisms through which nanoparticulate drug products could enable the transport of proteins and other large molecules across the BBB are not completely elucidated, but the most likely theory involves endocytosis followed by transcytosis (16). These mechanisms are assumed to influence the absorption rate through this physiological membrane.

Table 1. Comparison of CSF and Human Blood Plasma, Simulated CSF, and Simulated Body Fluid

\begin{tabular}{|c|c|c|c|c|}
\hline Composition & $\begin{array}{c}\text { Human } \\
\text { Blood } \\
\text { Plasma }\end{array}$ & $\begin{array}{c}\text { Simulated } \\
\text { Body Fluid }\end{array}$ & CSF & $\begin{array}{c}\text { Simualated } \\
\text { CSF }\end{array}$ \\
\hline $\mathrm{Na}^{+}$ & 142 & 142 & 154 & 150 \\
\hline $\mathrm{K}^{+}$ & 5 & 5 & 3.0 & 3.0 \\
\hline $\mathrm{Ca}^{2+}$ & 2.5 & 2.5 & 1.4 & 1.4 \\
\hline $\mathrm{Mg}^{2+}$ & 1.5 & 1.5 & 0.9 & 0.8 \\
\hline $\mathrm{Cl}^{-}$ & 103 & 148.8 & 136 & 155 \\
\hline $\mathrm{HCO}_{3-}$ & 27 & 4.2 & 24.1 & \\
\hline Protein (mg/dL) & 7000 & & 35 & \\
\hline Glucose (mg/dL) & 90 & & 60 & \\
\hline
\end{tabular}

Ionic concentrations expressed in $\mathrm{mM}$ unless otherwise noted. Bicarbonate is not added to the formula for artificial CSF as it converts to $\mathrm{CO}_{2}$ which can cause shifts in the $\mathrm{pH}$. The $\mathrm{pH}$ of human blood plasma, simulated body fluid, CSF, and simulated CSF was 7.25, 7.25, 7.3, and 7.3, respecively. CSF: cerebrospinal fluid.

Data from Refs. 47, 59, and 60.

\section{IN VITRO RELEASE TESTING FOR}

\section{NANOPARTICULATE DRUG PRODUCTS}

As nanoparticulate drug products gain more and more interest and transitioned from proof of concept to marketed products, it has become imperative to develop means to assess their in vitro performance, as it is part of the drug product approval and quality control requirements (25). Currently, there is no regulatory guidance for assessing in vitro drug release from parenterally administered nanoparticulate drug products. Compared to oral drug products, which benefit from welldefined regulatory standards and compendial apparatus, there is much space for improvement for testing of parenteral nanoparticulate drug formulations. Noticeable efforts have been made towards compensating by adapting classical methods and compendial apparatus for in vitro release testing methods for nanoparticulate dosage forms $(34,35)$.

\section{Preliminaries}

When developing an in vitro release test for nanosuspensions, general considerations described in the United States Pharmacopeia (USP) General Chapter $<1092>$ "The Dissolution Procedure: Development and Validation", may be taken into consideration regarding key parameters of the test, such as drug solubility, release medium, usage of filters, sampling, and sample preparation as well as for quantification and identification (36).

\section{In Vitro Dissolution/Drug Release Apparatus}

For testing the drug release from nanoparticulate products, compendial apparatus and modifications thereof, have been employed, as shown in Table $2(37,38)$.

In addition, compendial apparatus that may use finite volumes of medium can be based on stirring principles: USP 1 and USP 2, or flow-through principles: USP 4 in loop alignment, while USP 4 in open alignment is based on employment of an infinite volume of medium.

\section{USP 1 (basket method)}

To employ USP apparatus 1 with a basket configuration for testing the release from nanoparticles is difficult due to the structural features of the device. In vitro release test results provided with this setup were reported to have no discriminatory capacity when different formulations of cefuroxime axetil (CFA) or griseofulvin (GF) were tested, for which one possible explanation provided was the ability of nanoparticles to form agglomerations of different sizes and shapes inside the basket, which affects the wettability properties of the nanoparticles $(28,39)$.

Sievens-Figueroa et al. tested GF nanoparticles in the form of polymer strip-films, which were inserted inside the basket (39). Samples were taken at different time intervals and filtered using a syringe filter before being analyzed spectrophotometrically. Results showed a direct correlation between agitation speed and dissolution 
Table 2. Selected Methods Described for In Vitro Release Testing of Nano Sized Drug Carriers

\begin{tabular}{|c|c|c|c|c|c|}
\hline Drug Release Setting & Nano Formulation & Drug Substance & $\begin{array}{l}\text { Release } \\
\text { Medium }\end{array}$ & Medium Volume (mL) & Ref. \\
\hline \multicolumn{6}{|c|}{ Dialysis-Based Methods } \\
\hline $\begin{array}{c}\text { Continuous Flow Dialysis } \\
\text { Technique }\end{array}$ & BSA nanoparticle & 5-Fluorouracil & PBS (pH 7.4) & 40 & (61) \\
\hline Optimized USP 4 & Liposome & Dexamethasone & HEPES & 100 & (46) \\
\hline USP 1 and 4 & $\begin{array}{c}\text { Strip-film } \\
\text { nanosuspension }\end{array}$ & Griseofulvin & $0.54 \%(w / w)$ SDS & $\begin{array}{c}\text { 500-900 (USP 1); } 100 \\
\text { (USP 4) }\end{array}$ & (39) \\
\hline USP 2 & Nanosuspension & Indomethacin & $\begin{array}{c}\mathrm{HCl}(\mathrm{pH} 1.2), \text { phthalate buffer } \\
(\mathrm{pH} 5.0)\end{array}$ & 600 & (41) \\
\hline $\begin{array}{c}\text { Modified USP } 1 \\
\text { (+ membrane diffusion } \\
\text { cylinder })\end{array}$ & $\begin{array}{l}\text { PBCA nanoparticles, } \\
\text { gelatin B }\end{array}$ & $\begin{array}{l}\text { RIF, } \\
\text { MX }\end{array}$ & $\begin{array}{c}\text { PBS (pH 7.4), acetate buffer } \\
\quad(\mathrm{pH} 4.0), \mathrm{HCl}(\mathrm{pH} 1.2)\end{array}$ & 100 & (35) \\
\hline \multicolumn{6}{|c|}{ Sample and Separate Methods } \\
\hline USP 2 & $\begin{array}{l}\text { Lipidil } 145 \text { ONE, } \\
\text { Lipidil-Ter }\end{array}$ & Fenofibrate & $\begin{array}{c}\text { FaSSGF, FaSSIF, FaSSIF-V2, } \\
\text { FeSSIF-V2 }\end{array}$ & 500 & (27) \\
\hline $\begin{array}{l}\text { Ultracentrifugation; } \\
\text { Centrifugal Ultrafiltration; } \\
\text { Pressure Ultrafiltration }\end{array}$ & DOPC liposomes & Colistin & PBS (pH 7.4) & $0.5 ; 5 ; 50$ & (51) \\
\hline USP 1 and $2+$ Filtration & Nanoparticles & CFA & $0.1 \mathrm{M} \mathrm{HCl}+0.1 \mathrm{w} / \mathrm{v}$ SDS & 900 & (28) \\
\hline \multicolumn{6}{|c|}{ Continuous Flow Methods } \\
\hline USP 4 & Nanoparticles powder & CFA & $0.1 \mathrm{M} \mathrm{HCl}+0.1 \mathrm{w} / \mathrm{v}$ SDS & 900 & (28) \\
\hline
\end{tabular}

BSA, bovine serum albumine; PBS, phosphate buffered saline; HEPES, (4-(2-hydroxyethyl)-1-piperazineethanesulfonic acid), zwitterionic sulfonic acid buffering agent; SDS, sodium dodecyl sulphate; PBCA, polybutyl cyanoacrylate; FaSSGF, fasted state simulated gastric fluid; FaSSIF, FaSSIF-V2, FeSSIF-V2: three different types of fasted/fed state simulated intestinal fluid; DOPC, dioleoylphosphatidylcholine; USP: United States Pharmacopeia; HCl: hydrochloric acid; CFA: cefuroxime axetil; RIF, rifampicin; MX: moxifloxican $\mathrm{HCl}$

rate. However, situations where the films attached to the walls of the basket or formation of nanoparticle agglomerations due to the low sheer forces inside the basket were encountered. When GF nanoparticles were tested against GF microparticles at a high agitation speed, the dissolution profiles were similar, which renders the method not suitable for discriminating based on particle size (39). Heng et al. encountered similar challenges when testing release from processed amorphous nanoparticles and the unprocessed crystalline form of CFA using the basket method. The nanoparticles submerged with the basket into the medium were observed floating at the surface of the medium (28). This highlights the containment issue related to nanoparticles when using the basket method. Also, a fast initial dissolution rate followed by a slower dissolution rate was observed, most likely due to the agglomeration of the nanoparticles (28).

\section{USP 1 modified (cylinder method)}

Gao et al. describe using USP 1 in conjunction with a membrane diffusion cylinder, represented by a flat-bottom cell with the opening covered by a dialysis membrane, to test different gelatin and polybutyl cyanoacrylate (PBCA) nanoparticles formulations, loaded with rifampicin (RIF) and moxifloxacin hydrochloride (MX). The in vitro release test was conducted under forced (enzymatic degradation) and non-forced conditions for the gelatin nanoparticles, which lead to a faster release rate in the presence of the enzymes that only the modified setup was able to detect. In addition, the modified cylinder method was able to identify the different release mechanisms of RIF and MX (35).

\section{USP 2 (paddle method)}

The paddle method encounters the same artifacts, aggregation, agglomeration, and poor wettability of the nanoparticles at different rotation speeds and volumes, as with the use of USP 1 , rendering it difficult to assess the true release rate $(28,34,40)$. The employment of non-sink conditions were reported to be preferable to 
sink conditions, as observed by Liu et al. when testing three different particle sizes of indomethacin nanosuspensions (41).

A study published by Yang et al. tested in vitro release from nanoscale drug depots in the form of core-shell fibers using the paddle method (42). The core-shell fiberbased depots containing ferulic acid (FA) in a cellulose acetate (CA) matrix were prepared using a modified triaxial electrospinning process. When tested in $900 \mathrm{~mL}$ phosphate buffered saline at $37 \pm 1^{\circ} \mathrm{C}$ and $50 \mathrm{rpm}$, over 36 hours, the core-shell depots showed no initial burst release effect and the drug release process may be considered zero-order release, with a correlation coefficient $(R)$ of 0.9868 , as opposed to monolithic CA/FA fibers (CA nanofibers from single-fluid electrospinning), for which a significant initial burst release was noticed (42).

\section{USP 2 modified (dispersion releaser)}

Developed by Wacker, the device is comprised of a sample holder cell (donor compartment) equipped with a paddle stirrer and the acceptor compartment, represented by a mini or a full size dissolution vessel, as shown in Figure 1 (43). The sample holder is a hollow cylinder framework on which the dialysis membrane is fixed with O-rings (rubber rings). Due to the unique feature of having an outside stirrer (in the acceptor compartment) as well as an inside stirrer (inside the donor compartment), using the dispersion releaser showed improved hydrodynamics compared with regular dialysis setups. Experimental results proved that the dispersion releaser is able to discriminate between changes in the formulation and is suitable for testing nanoparticulate drug products (44).

\section{USP 4 (flow-through cell in loop alignment)}

The flow-through apparatus can be used as a loop system when the medium is continuously recirculated through the cell, which makes it suitable for tests where low volumes of medium are required $(13,43)$. For testing nanoparticulate drug products, this setup was shown to discriminate between different formulations (39). Furthermore, a higher dissolution rate from the nanoparticulate drug product was noticed when compared with the drug substance powder in its unprocessed form, most likely due to an increased surface area and solubility, as shown by Heng et al. (28). Also, the containment issues encountered with the basket method were overcome, as the nanoparticles were retained inside the flowthrough cell (28). Thus, USP 4 may be suitable to detect differences in the dissolution behaviour of nanoparticles with different sizes (28).

\section{USP 4 (flow-through cell in open alignment)}

USP 4 when aligned as an open system, provides continuously fresh medium passing through the cell (infinite volume), which requires large volumes of medium $(13,43)$. As a result, the large total volume of media may contain small masses of drug, which may limit the analytical quantification, or even render it impossible.

\section{USP 4 modified with dialysis adapter}

The modified USP 4 setup is comprised of a dialysis adapter placed inside the sample cell of the flow through apparatus in upright position (schematic detailed in Fig. 2) (46). The design of the adapter, described by Bhardwaj and Burgess, consists in a cylindrical structure on which the dialysis membrane is mounted and sealed at the ends with O-rings (similar to the dispersion releaser cell). The modified USP 4 was applied for characterization of suspensions and liposomes containing dexamethasone (46). Compared to classic stirred beaker dialysis setups, such as dialysis sac and reverse dialysis, this modified USP 4 setup for testing in vitro release of nanosuspensions and liposomes was described as being superior in terms of discriminatory ability and robustness (46).

\section{Release Medium}

When selecting the release medium, the same considerations as for conventional oral dosage forms apply. Primarily, the route of administration, drug substance solubility and stability, and site of action will influence the choice of volume and composition of the medium. Secondly, technical aspects have to be considered such as in vitro solubility, stability of the drug release testing medium and of the drug in solution, and stability of the whole system over the time span required for the test run. Eventually the accuracy and precision of the method determine its suitability showing differences in release kinetics and hence, the similarities. For parenteral nanosuspensions, selection of the release medium usually starts with artificial human blood plasma (47). However, artificial synovial fluid and simulated CSF have also been employed $(47,48)$. For robust testing, due to the complex nature of the medium, the composition should be simplified while keeping the physiological parameters constant, such as $\mathrm{pH} 7.4$ and osmotic pressure $285 \mathrm{mOsm} / \mathrm{kg}$ for blood plasma (49). Although sink conditions are preferred, in vitro release test methods for nanoparticulate drug products may also be performed under non-sink conditions (41). Agitation rate as well as the presence of solubilizers (surfactants, cyclodextrins) can have an impact on the release kinetics $(35,49)$. The volume of the medium can be adapted based 


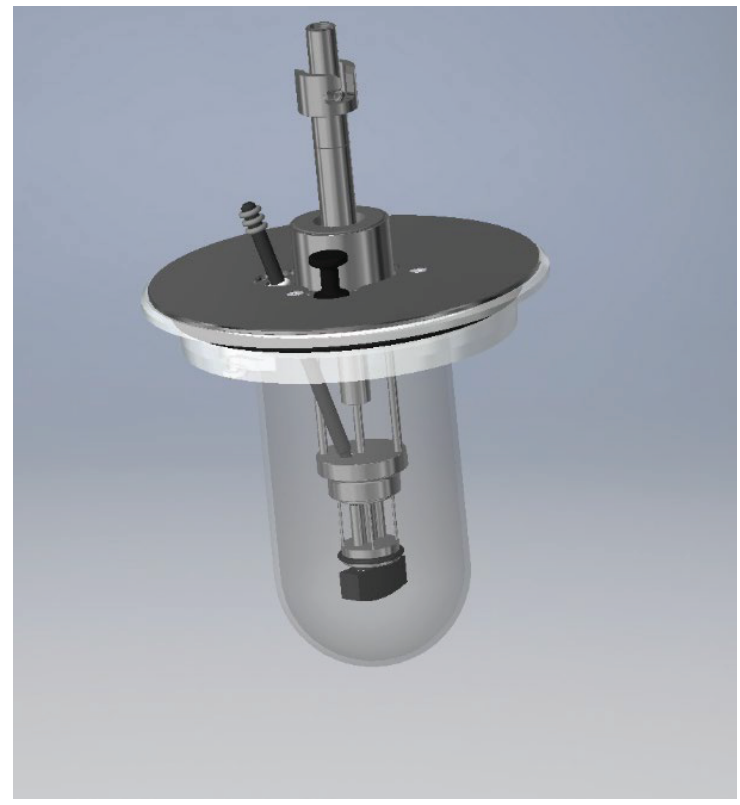

Figure 1. 3D schematic of the dispersion releaser.

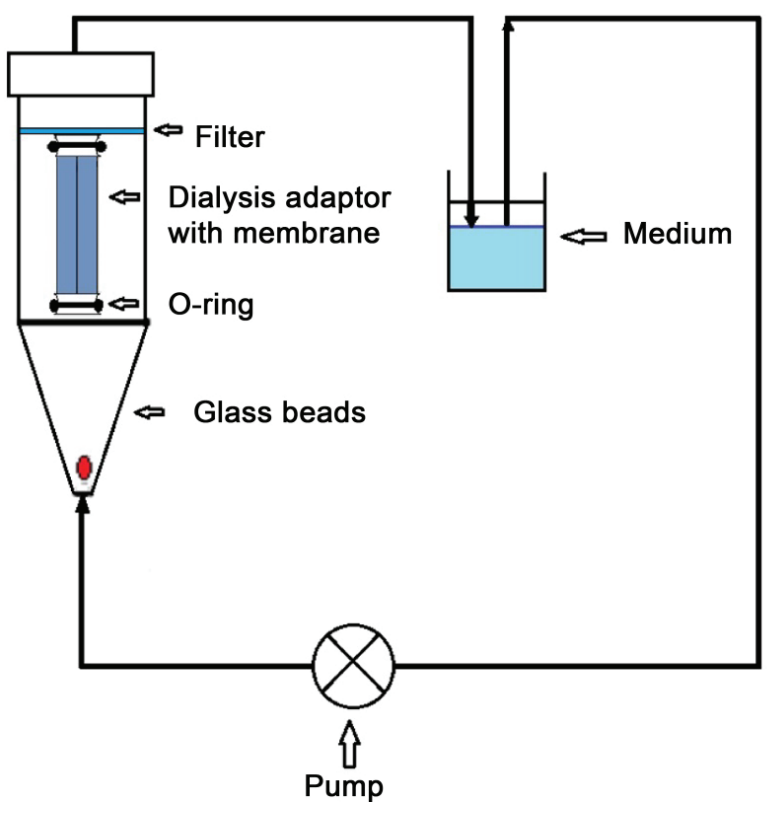

Figure 2. Schematic of the dialysis adapter design: placement of the adapter in USP apparatus 4 in upright position inside the sample cell. on the setup. Volumes varying from 1 to $900 \mathrm{~mL}$ have been reported for the sampling and separating methods as compared to dialysis methods, where the volumes for the donor compartment range from $100 \mu \mathrm{L}$ up to $40 \mathrm{~mL}$ and up to $900 \mathrm{~mL}$ for the acceptor compartment $(27,43)$.

\section{Separation Techniques}

When assessing drug release from nanoparticulate dosage forms, the main concern is to quantify the released moiety of drug substance in the presence of the carrier bound moiety, which may require physical separation. Physical separation methods based on filtration and centrifugation are used besides physicochemical methods (solid phase extraction [SPE]) $(26,50-52)$.

The versatility of the sample and separate methods is overcome by the difficulty of the separation step and lack of precision. Dialysis membrane-based methods compensate for this drawback by integrating the separation step into the release test, with the necessity to determine the diffusion rate of the drug in solution through the dialysis membrane $(13,26)$.

\section{Use of Membranes}

The separation process takes place during the release test and can be achieved through various methods: dialysis bag, side-by-side diffusion cell method, reverse dialysis, and the latest, the dispersion releaser with the sample holder cell which can be attached to the basket/paddle shaft of the compendial USP 1 or 2 apparatus $(44,45)$. The principle is to disperse the nanoparticulate formulation in the release medium, in a donor compartment, from where the free drug substance diffuses through a membrane in the acceptor compartment. This setup requires that the drug release rate in the donor compartment be slower than the membrane passage (44). In most cases (dialysis bag, dispersion releaser), the nanosuspension is inserted in the smaller closed inner compartment and samples are collected from the larger outer compartment. With the reverse dialysis and side-by-side dialysis method, the nanosuspension is applied in thelarger outer compartment and samples are withdrawn from the inner compartment $(13,39,45)$. Additionally, the reversed dialysis method mimics the in vivo conditions for parenteral (and also oral) nanoparticulate drug products, as infinite dilution occurs once they are inserted, respectively administered (54). Compared to sequential sampling and separating techniques, dialysis methods are more convenient, since separation is occurring concomitantly to the drug release. The choice of membrane (in terms of material and molecular weight cut off) is of major impact (45). Computation of the release kinetics may need additional membrane permeation experiments for drug solutions, combined with mathematical deconvolution if needed (26).

\section{Sample and Separate Method (Tube Method)}

Compared to the use of dialysis membranes as a separator inside the dissolution vessel, sample and separate 
methods require separation of the released drug from the bound fraction after the sampling. Separation of the two fractions, bound and unbound drug, can be done by means of filtration, ultracentrifugation, ultrafiltration, $\mathrm{SPE}$, or asymmetrical flow field flow fractionation (AF4) $(50,54)$. Filtration is a common technique, easy to perform and reproducible; however, special attention must be paid to the filtering material. Firstly, due to adsorption effects and/or drug-filter material or membrane interactions; secondly, due to the tendency of nanoparticles to form aggregates that could block the filters (36). Another method, pressure ultrafiltration, involves the use of low hydrostatic pressure on the particles. This technique provides fast and reliable results and may have applications in quality control (51). Preparative ultra-centrifugation uses high centrifugal forces over a long period of time, which determines the sedimentation of the nanoparticles. Limitations of this method have been mentioned by Wallace et al. and Beyer et al. for nanoparticles smaller than $100 \mathrm{~nm}$, among which incomplete separation and the potential to create artifacts by continuing release from the carrier after sampling, due to the high shear forces applied $(50,55)$. SPE uses a solid (stationary) phase and a liquid (mobile) phase to separate the dissolved drug substance from the nano carrier. Compared to filtration and ultracentrifugation, the integrity of the particles is not affected. However, for an increased efficiency, the drug substance and the carrier matrix must have different affinities for the SP, which could represent a limitation for this method (52).

The versatility of the sample and separate method is represented by the possibility to choose between different setups: USP 1, USP 2, or vials; different volumes of dissolution/release medium: from 1 to $900 \mathrm{~mL}$; or type of agitation of the medium: orbital shakers or magnetic stirrers $(27,50)$.

\section{In Situ Quantification not Requiring Sampling or Separation}

In situ measurements, which are performed directly in the bulk medium during the in vitro release test, render sampling and physical separation redundant. The prerequisite is that the analytical method can be performed in the dissolution vessel. Applications based on dynamic light scattering (DLS), UV-VIS detection, fluorescence, ion-selective electrode (potentiometry), and calorimetric methods are described $(40,56)$. However, the analytical properties of the drug substance are primarily determining the choice of the analytical finish. These non-consumptive procedures usually allow high numbers of measurement time points, with the benefit of a high resolution of the release kinetics.

A particular case is represented by the use of in situ fiber optics combined with derivative spectroscopy as described by Guillot et al. for PLGA-based nanoparticles (58). The setup comprised a UV-VIS spectrophotometer connected to a compendial basket apparatus by fiber optics. The approach is using derivative UVspectrophotometry to level out matrix and medium interferences and provides real-time data by in situ monitoring of the drug release kinetics with optical fibers. This method is suitable for automation and can also be used for small volumes of medium. Limitations to this method are the degree of turbidity and UV absorption characteristics of the analyte. This combined method allows quantification of the dissolved drug substance in the presence of the moiety of active ingredient, which is still bound to the nanoparticulate carrier, and has been validated for GMP purposes (58). Avoiding sampling, separation, and liquid handling as well as human error and the overall precision of fiber optics combined with derivative spectroscopy may be superior to the classical sample-and-separate methodology.

\section{Duration of the Test}

Parenteral nanoparticulate drug products are usually designed for long-term controlled drug release over periods of time ranging from days to weeks. This is mostly the case for subcutaneous and intramuscular routes, when a depot may be involved, but not excluding the IV route when the rate of release may be controlled by the carrier through the formulation technology. A real-time in vitro release test may not always be a convenient option from the stability of the in vitro setup point of view. Therefore, accelerated tests may be preferred. For this purpose, parameters such as temperature, agitation, or composition of media may be modified to accelerate the drug release, provided that only the rate changes but the mechanism of the release remains unaltered (37).

\section{CONCLUSION}

The basis of in vitro drug release testing methods of nanoparticulate drugs is considered by the traditional approach with sampling and separating either by filtration or using dialysis membranes. Finite volumes are still preferred to continuous flow setups. With a look to the future, suitable in vitro release test for nanoparticulate drug products are defined by their use as so-called "regulatory approved" methods, which means being used for batch release testing. Precision is one of 
the challenges for a discriminative but analytically valid method. In that regard, methods such as the dispersion releaser or the use of optical fibers, hold great promise for the future.

A standardized in vitro release test is the prerequisite for meaningful nanoparticulate dosage form development and performance testing. With an in vitro-in vivocorrelation being established, changes of the in vivo performance may be predicted by in vitro testing. With the limitations on how to access the in vivo release kinetics, mathematical in silico modelling may need to be applied to predict the in vivo release rate based on in vitro data. In addition, if the site of action is separated by barriers, introducing pharmacokinetic steps, new approaches are needed. The classical approach based on bioavailability, with the rate and amount of drug available in the systemic circulation as the surrogate for therapeutic effect, would need to be extended further. With that deficiency, the need of meaningful in vitro drug release testing methods gains great importance.

\section{CONFLICT OF INTEREST}

The authors disclosed no conflicts of interest related to this article.

\section{REFERENCES}

1. Gulati, N.; Gupta, H. Parenteral Drug Delivery: A Review. Recent Pat. Drug Deliv. Formul. 2011, 5, 133-145. DOI:10.2174/187221111795471391.

2. Burgess, D. J.; Clark, B. C.; Hampson-Carlin, M. J.; Shah, P. Critical Quality and Performance Parameters for Modified-Release Parenteral Dosage Form. Pharmacopeial Forum 2005, 31, 17451748.

3. Ghadge, T. A.; Chavare, S. D.; Shruti, K. A Review on Parentral Implants. IJRRPAS 2014, 4 (2), 1056-1072.

4. Ventola, C. L. Progress in Nanomedicine: Approved and Investigational Nanodrugs. 2019, 42 (January), 1-5. DOI:10.1016/j.psychres.2007.07.030.

5. Schichtel, J. Determination of the Dissolution Behavior of Celecoxib-Eudragit E 100-Nanoparticles Using CrossFlow Filtration. Doctoral Dissertation [Online], Johannes Gutenberg-Universität, Mainz, 2017 DOI:urn:nbn:de:hebis:77diss-1000009033.

6. Türeli, A. E. Nanoparticle Preparation Process Using Novel Microjet Reactor Technology for Enhancing Dissolution Rates of Poorly Water Soluble Drugs. Doctoral Dissertation [Online], Johannes Gutenberg-Universität, Mainz, 2015 DOI:urn:nbn:de:hebis:77-40453.

7. Kingsley, J. D.; Dou, H.; Morehead, J.; Rabinow, B.; Gendelman, H. E.; Destache, C. J. Nanotechnology: A Focus on Nanoparticles as a Drug Delivery System. J. Neuroimmune Pharmacol. 2006, 1 (3), 340-350. DOI:10.1007/s11481-006-9032-4.
8. Kreuter, J. Evaluation of nanoparticles as drug-delivery systems. III. Materials, stability, toxicity, possibilities of targeting, and use; Pharm Acta Helv, 1983.

9. Shen, J.; Burgess, D. J. Accelerated in Vitro Release Testing Methods for Extended Release Parenteral Dosage Forms. J. Pharm. Pharmacol. 2012, 64 (7), 986-996. DOI:10.1111/j.20427158.2012.01482.x.

10. Bhatia, S. Nanoparticles Types, Classification, Characterization, Fabrication Methods and Drug Delivery Applications. In Natural Polymer Drug Delivery Systems; Springer International Publishing, 2016; pp 33-93 DOI:10.1007/978-3-319-41129-3.

11. Kamensky, A. A.; Kondrasheva, I. G.; Severin, E. S.; Guseva, A. A.; Gambaryan, P. Y. Increasing the Efficiency of Parkinson's Disease Treatment Using a Poly(Lactic-Co-Glycolic Acid) (PLGA) Based L-DOPA Delivery System. Exp. Neurobiol. 2014, 23 (3), 246-252. DOI:10.5607/en.2014.23.3.246.

12. Barenholz, Y. Doxil ${ }^{\circledR}$ - The First FDA-Approved Nano-Drug: Lessons Learned. J. Control. Release 2012, 160 (2), 117-134. DOI:10.1016/j.jconrel.2012.03.020.

13. D'Souza, S. A Review of In Vitro Drug Release Test Methods for Nano-Sized Dosage Forms . Adv. Pharm. 2014, 2014, 1-12. DOI:10.1155/2014/304757.

14. Wagner, S.; Zensi, A.; Wien, S. L.; Tschickardt, S. E.; Maier, W.; Vogel, T.; Worek, F.; Pietrzik, C. U.; Kreuter, J.; von Briesen, H. Uptake Mechanism of ApoE-Modified Nanoparticles on Brain Capillary Endothelial Cells as a Blood-Brain Barrier Model. PLoS One 2012, 7 (3), 3-9. DOI:10.1371/journal.pone.0032568.

15. Feczkó, T.; Tóth, J.; Dósa, G.; Gyenis, J. Influence of Process Conditions on the Mean Size of PLGA Nanoparticles. Chem. Eng. Process. Process Intensif. 2011, 50 (8), 846-853. DOI:10.1016/j. cep.2011.05.006.

16. Kreuter, J. Drug Delivery to the Central Nervous System by Polymeric Nanoparticles: What Do We Know? Adv. Drug Deliv. Rev. 2014, 71, 2-14. DOI:10.1016/j.addr.2013.08.008.

17. Gelperina, S.; Maksimenko, O.; Khalansky, A.; Vanchugova, L.; Shipulo, E.; Abbasova, K.; Berdiev, R.; Wohlfart, S.; Chepurnova, N.; Kreuter, J. Drug Delivery to the Brain Using Surfactant-Coated Poly(Lactide-Co-Glycolide) Nanoparticles: Influence of the Formulation Parameters. Eur. J. Pharm. Biopharm. 2010, 74 (2), 157-163. DOI:10.1016/j.ejpb.2009.09.003.

18. Commission Recommendation of 18 October on the Definition of Nanomaterial (2011/696/EU). Off. J. Eur. Union 2011, No. 275, 38-40.

19. Guidance for Industry: Considering Whether an FDA-Regulated Product Involves the Application of Nanotechnology Contains Nonbinding Recommendations. U.S. Department of Health and Human Services Food and Drug Administration Office of the Commissioner 2014, pp 1-14.

20. GB/T 19619-2004 Terminology for Nanomaterials. Standard Administration of China, 2004.

21. FDA/CDER. Drug Products, Including Biological Products, That Contain Nanomaterials - Guidance for Industry. In FDA; 2017 DOI:10.1002/jgm. 
22. Jeevanandam, J.; Barhoum, A.; Chan, Y. S.; Dufresne, A.; Danquah, M. K. Review on Nanoparticles and Nanostructured Materials: History, Sources, Toxicity and Regulations. Beilstein J. Nanotechnol. 2018, 9(1), 1050-1074. DOI:10.3762/bjnano.9.98.

23. Papaluca, M. First International Scientific Workshop on Nanomedicines. In European Medicines Agency's workshop on nanomedicines; European Medicines Agency: London, September 2-3, 2010.

24. Pita, R.; Ehmann, F.; Papaluca, M. Nanomedicines in the EU-Regulatory Overview. AAPS J. 2016, 18 (6), 1576-1582. DOI:10.1208/s12248-016-9967-1.

25. Siewert, M.; Dressmann, J.; Brown, C. K.; Shah, V. P. FIP / AAPS Guidelines to Dissolution / in Vitro Release Testing of Novel / Special Dosage Forms. AAPS PharmSciTech 2003, 4 (1), 1-10. DOI:10.1208/pt040107.

26. Janas, C.; Mast, M.-P.; Kirsamer, L.; Angioni, C.; Gao, F.; Mäntele, W.; Dressman, J.; Wacker, M. G. The Dispersion Releaser Technology Is an Effective Method for Testing Drug Release from Nanosized Drug Carriers. Eur. J. Pharm. Biopharm. 2017, 115, 73-83. DOI:10.1016/j.ejpb.2017.02.006.

27. Juenemann, D.; Jantratid, E.; Wagner, C.; Reppas, C.; Vertzoni, M.; Dressman, J. B. Biorelevant in Vitro Dissolution Testing of Products Containing Micronized or Nanosized Fenofibrate with a View to Predicting Plasma Profiles. Eur. J. Pharm. Biopharm. 2011, 77 (2), 257-264. DOI:10.1016/j.ejpb.2010.10.012.

28. Heng, D.; Cutler, D. J.; Chan, H. K.; Yun, J.; Raper, J. A. What Is a Suitable Dissolution Method for Drug Nanoparticles? Pharm. Res. 2008, 25 (7), 1696-1701. DOI:10.1007/s11095-008-9560-0.

29. Mohanraj, V. J.; Chen, Y. Nanoparticles - A Review. Trop. J. Pharm. Res. 2006, 5 (1), 561-573. DOI:10.4314/tjpr.v5i1.14634.

30. Salmaso, S.; Caliceti, P. Stealth Properties to Improve Therapeutic Efficacy of Drug Nanocarriers. J. Drug Deliv. 2013, 2013, 1-19. DOI:10.1155/2013/374252.

31. Gulyaev, A. E.; Svetlana, G. E.; Skidan, I. N.; Antropov, A. S.; Kivman, G. Y.; Kreuter, J. Significant Transport of Doxorubicin into the Brain with Polysorbate 80-Coated Nanoparticles. Pharm. Res. 1999, 16 (10), 1564-1569. DOI:10.1023/A:1018983904537.

32. Gabizon, A.; Shmeeda, H.; Barenholz, Y. Pharmacokinetics of Pegylated Liposomal Doxorubicin: Review of Animal and Human Studies. Clin. Pharmacokinet. 2003, 42 (5), 419-436. DOI:10.2165/00003088-200342050-00002.

33. Serlin, Y.; Shelef, I.; Knyazer, B.; Friedman, A. Anatomy and Physiology of the Blood-Brain Barrier. Semin. Cell Dev. Biol. 2015, 38, 2-6. DOI:10.1016/j.semcdb.2015.01.002.

34. Nicklasson, M.; Orbe, A.; Lindberg, J.; Borgå, B.; Magnusson, A. B.; Nilsson, G.; Ahlgren, R.; Jacobsen, L. A Collaborative Study of the in Vitro Dissolution of Phenacetin Crystals Comparing the Flow through Method with the USP Paddle Method. Int. J. Pharm. 1991, 69 (3), 255-264. DOI:10.1016/0378-5173(91)90367-W.

35. Gao, Y.; Zuo, J.; Bou-Chacra, N.; Pinto, T. de J. A.; Clas, S.D.; Walker, R. B.; Löbenberg, R. In Vitro Release Kinetics of
Antituberculosis Drugs from Nanoparticles Assessed Using a Modified Dissolution Apparatus. Biomed Res. Int. 2013, 2013, 1-9. DOI:10.1155/2013/136590.

36. <1092> The Dissolution Procedure: Development and Validation. In United States Pharmacopeia and National Formulary USP 41-NF 36; The United States Pharmacopeial Convention, Inc.: Rockville, MD, 2018; pp 7178-7198.

37. D'Souza, S. S.; DeLuca, P. P. Methods to Assess in Vitro Drug Release from Injectable Polymeric Particulate Systems. Pharm. Res. 2006, 23 (3), 460-474. DOI:10.1007/s11095-005-9397-8.

38. Gray, V.; Cady, S.; Curran, D.; Demuth, J.; Eradiri, O.; Hussain, M.; Krämer, J.; Shabushnig, J.; Stippler, E. In Vitro Release Test Methods for Drug Formulations for Parenteral Applications. Dissolution Technol. 2018, 25 (4), 8-13. DOI:10.14227/ DT250418P8.

39. Sievens-Figueroa, L.; Pandya, NatashaBhakay, Bhakay, A.; Keyvan, G.; Michniak-Kohn, B.; Bilgili, E.; Davé, R. N. Using USP I and USP IV for Discriminating Dissolution Rates of Nanoand Microparticle-Loaded Pharmaceutical Strip-Films. AAPS PharmSciTech 2012, 13 (4), 1473-1482. DOI:10.1208/s12249012-9875-3.

40. Anhalt, K.; Geissler, S.; Harms, M.; Weigandt, M.; Fricker, G. Development of a New Method to Assess Nanocrystal Dissolution Based on Light Scattering. Pharm. Res. 2012, 29 (10), 2887-2901. DOI:10.1007/s11095-012-0795-4.

41. Liu, P.; De Wulf, O.; Laru, J.; Heikkilä, T.; van Veen, B.; Kiesvaara, J.; Hirvonen, J.; Peltonen, L.; Laaksonen, T. Dissolution Studies of Poorly Soluble Drug Nanosuspensions in Non-Sink Conditions. AAPS PharmSciTech 2013, 14 (2), 748-756. DOI:10.1208/ s12249-013-9960-2.

42. Yang, G. Z.; Li, J. J.; Yu, D. G.; He, M. F.; Yang, J. H.; Williams, G. R. Nanosized Sustained-Release Drug Depots Fabricated Using Modified Tri-Axial Electrospinning. Acta Biomater. 2017, 53, 233-241. DOI:10.1016/j.actbio.2017.01.069.

43. Wacker, M. G. Challenges in the Drug Release Testing of NextGeneration Nanomedicines - What Do We Know? Mater. Today Proc. 2017, 4, S214-S217. DOI:10.1016/j.matpr.2017.09.189.

44. Jung, F.; Nothnagel, L.; Gao, F.; Thurn, M.; Vogel, V.; Wacker, M. G. A Comparison of Two Biorelevant in Vitro Drug Release Methods for Nanotherapeutics Based on Advanced PhysiologicallyBased Pharmacokinetic Modelling, Elsevier, 2018, Vol. 127 DOI:10.1016/j.ejpb.2018.03.010.

45. Nothnagel, L.; Wacker, M. G. How to Measure Release from Nanosized Carriers? Eur. J. Pharm. Sci. 2018, 120 (May), 199211. DOI:10.1016/j.ejps.2018.05.004.

46. Bhardwaj, U.; Burgess, D. J. A Novel USP Apparatus 4 Based Release Testing Method for Dispersed Systems. Int. J. Pharm. 2010, 388 (1-2), 287-294. DOI:10.1016/j.ijpharm.2010.01.009.

47. Marques, M. R. C.; Loebenberg, R.; Almukainzi, M. Simulated Biologic Fluids with Possible Application in Dissolution Testing. Dissolution Technol. 2011, No. August, 15-28. DOI:10.14227/ DT180311P15. 
48. Kumar, P.; Choonara, Y. E.; du Toit, L. C.; Singh, N.; Pillay, V. In Vitro and in Silico Analyses of Nicotine Release from a GelisphereLoaded Compressed Polymeric Matrix for Potential Parkinson's Disease Interventions. Pharmaceutics 2018, 10 (4), 1-17. DOI:10.3390/pharmaceutics10040233.

49. Delplace, C.; Kreye, F.; Klose, D.; Danède, F.; Descamps, M.; Siepmann, J.; Siepmann, F. Impact of the Experimental Conditions on Drug Release from Parenteral Depot Systems: From Negligible to Significant. Int. J. Pharm. 2012, 432 (1-2), 11-22. DOI:10.1016/j.ijpharm.2012.04.053.

50. Jana, U.; Mohanty, A. K.; Pal, S. L.; Manna, P. K.; Mohanta, G. P. Felodipine Loaded PLGA Nanoparticles: Preparation, Physicochemical Characterization and in Vivo Toxicity Study. Nano Converg. 2014, 1 (1), 31. DOI:10.1186/s40580-014-00315.

51. Wallace, S. J.; Li, J.; Nation, R. L.; Boyd, B. J. Encapsulation and Release Methodology. Dug Deliv Transl Res 2012, 2 (4), 284-292. DOI:10.1007/s13346-012-0064-4.

52. Guillot, A.; Couffin, A.; Sejean, X.; Navarro, F.; Limberger, M.; Lehr, C. Solid Phase Extraction as an Innovative Separation Method for Measuring Free and Entrapped Drug in Lipid Nanoparticles. Pharm Res. 2015, 32, 3999-4009. DOI:10.1007/s11095-0151761-8.

53. Guillot, A.; Limberge, M.; Krämer, J.; Lehr, C. M. In Situ Drug Release Monitoring with a Fiber-Optic System: Overcoming Matrix Interferences Using Derivative Spectrophotometry. Dissolution Technol. 2013, 20 (2), 15-19. DOI:10.14227/ DT200213P15.

54. Chidambaram, N.; Burgess, D. J. A Novel in Vitro Release Method for Submicron-Sized Dispersed Systems. AAPS PharmSci 2003, 1 (3), 32-40. DOI:10.1208/ps010311.
55. Jünemann, D.; Dressman, J. Analytical Methods for Dissolution Testing of Nanosized Drugs. J. Pharm. Pharmacol. 2012, 64 (7), 931-943. DOI:10.1111/j.2042-7158.2012.01520.x.

56. Beyer, S.; Xie, L.; Gräfe, S.; Vogel, V.; Dietrich, K.; Wiehe, A.; Albrecht, V.; Mäntele, W.; Wacker, M. G. Bridging Laboratory and Large Scale Production: Preparation and In Vitro-Evaluation of Photosensitizer-Loaded Nanocarrier Devices for Targeted Drug Delivery. Pharm. Res. 2014, 32 (5), 1714-1726. DOI:10.1007/ s11095-014-1569-y.

57. Juenemann, D.; Bohets, H.; Ozdemir, M.; De Maesschalck, R.; Vanhoutte, K.; Peeters, K.; Nagels, L.; Dressman, J. B. Online Monitoring of Dissolution Tests Using Dedicated Potentiometric Sensors in Biorelevant Media. Eur. J. Pharm. Biopharm. 2011, 78 (1), 158-165. DOI:10.1016/j.ejpb.2010.12.014.

58. Guillot, A. Analyzing Drug Load and Release from Pharmaceutical Nanocarriers for the Treatment of Inflammatory Bowel Diseases. Doctoral Dissertation [Online], Universität des Saarlandes, Saarbrücken, Germany, 2014 DOI:10.22028/D291-23051.

59. Davson, H. Physiology of the Cerebrospinal Fluid, Volume III, 2nd ed.; J. \& A. Churchill, Ltd.: London, 1967.

60. Altman, P. L.; Dittmer, D. S. Biology Data Book, Volume III, 2nd ed.; Federation of American Societies for Experimental Biology: Washington D.C., 1974.

61. Maghsoudi, A.; Shojaosadati, S. A.; Vasheghani Farahani, E. 5-Fluorouracil-Loaded BSA Nanoparticles: Formulation Optimization and In Vitro Release Study. AAPS PharmSciTech 2008, 9 (4), 1092-1096. DOI:10.1208/s12249-008-9146-5. 